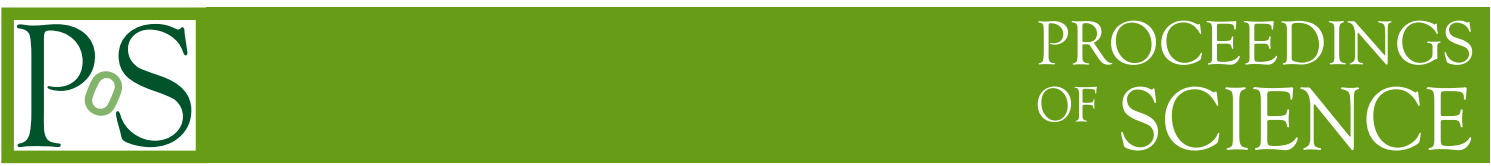

\title{
Charm (-onium) physics at PANDA
}

\section{Frank Nerling, on behalf of the PANDA Collaboration}

Helmholtz Forschungsakademie Hessen für FAIR, GSI Helmholtzzentrum für Schwerionenforschung, Campus Frankfurt, Max-von-Laue Strasse 12, Frankfurt am Main, Germany

E-mail: f.nerling@gsi.de

The new Facility for Antiproton and Ion Research (FAIR) is under construction at GSI in Darmstadt (Germany). The PANDA experiment represents the central part of the hadron physics programme, addressing a wide range of topics in hadron structure and spectroscopy, most notably in the charm sector. The field of hadron spectroscopy has gained new momentum by the discovery of various new hadronic resonances especially in the charmonium sector over the past two decades. The nature of many of the so-called charmonium-like exotic XYZ states are, however, not yet understood. Precise measurements of hadron masses and widths are mandatory to sort out different theoretical models and clarify the nature of these new states. One example is the $X(3872)$ - although being the first of the new charmonium-like states discovered since 2003, the nature of this state is still not understood. Via $p \bar{p}$ annihilation, such XYZ states can be produced at PANDA in direct formation, allowing for a precise resonance energy scan. Using the example of the $X(3872)$, we quantified the expected sensitivity of energy scans of narrow resonances and how well we can distinguish between models that turn out to be indistinguishable from the LHCb data. Also, PANDA will be feasible to complete the XYZ multiplets by strange partner and high-spin states. Moreover, charmed hybrids as well as open charm $D_{s}$ states will be searched for and investigated at PANDA.

\footnotetext{
*** 10th International Workshop on Charm Physics (CHARM2020), ***

*** 31 May - 4 June, 2021 ***

*** Mexico City, Mexico - Online ***
} 


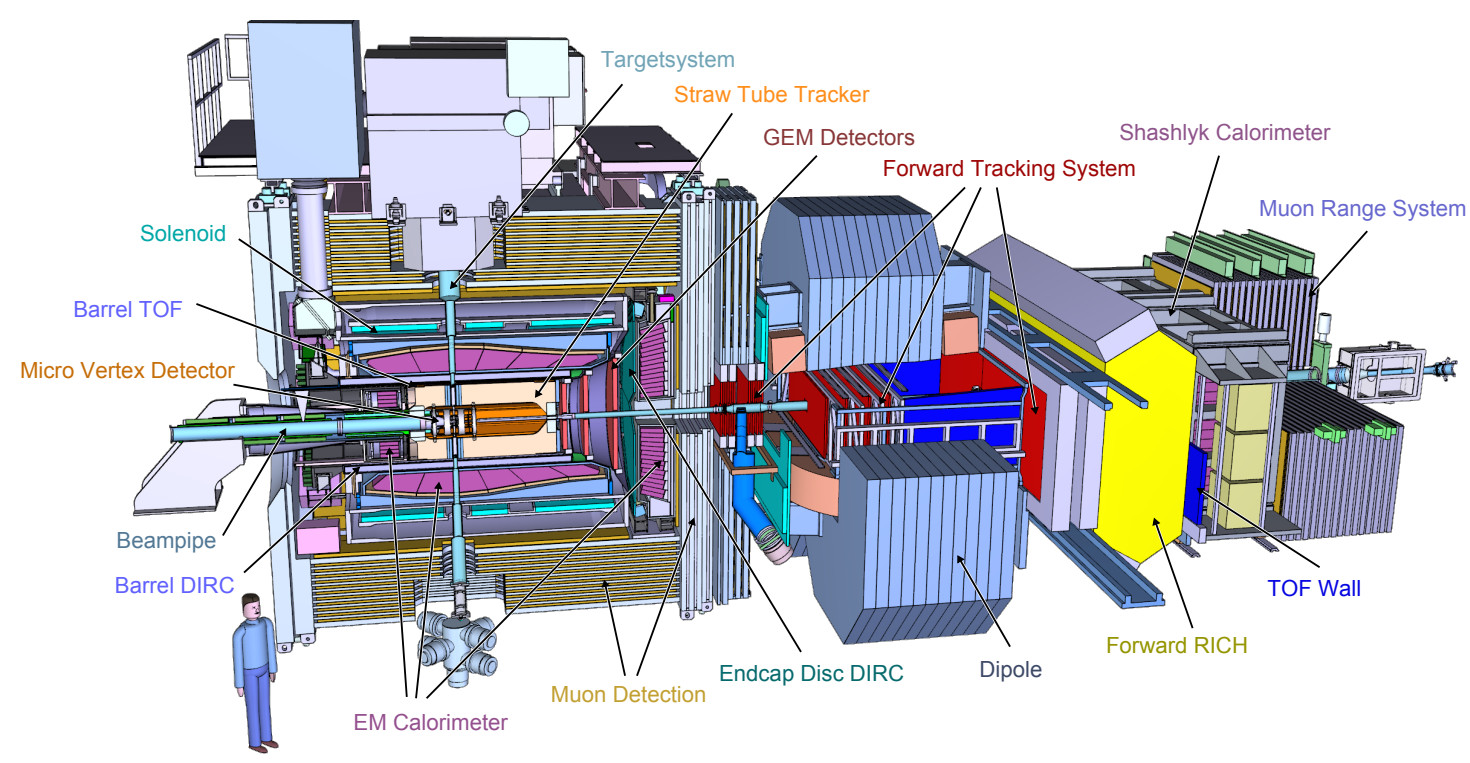

Figure 1: The proposed PANDA fixed-target experimental setup consists of a barrel spectrometer surrounding the target region and a forward spectrometer.

\section{Introduction}

Bound states of the strong interaction are described by Quantum Chromodynamics (QCD). In the charmonium region, they can be described quite successfully based on potential models. Beneath the open-charm threshold, all the predicted states have been observed with the expected properties and there is excellent agreement between model calculations and experiment. Above the opencharm threshold, however, there are still many predicted states that have not yet been discovered, and more than 20 unexpected states have surprisingly been observed since 2003. Famous examples of these so-called (exotic) charmonium-like "XYZ" states are the $X(3872)$ observed by Belle [1] in 2003, the $Y$ (4260) and $Y$ (4360), both discovered by BaBar using initial state radiation (ISR) [2, 3], and the charged state $Z_{\mathrm{c}}(3900)^{ \pm}$discovered by BESIII [4], and shortly after confirmed by Belle [5], that, due to the mass in combination with the electrical charge, is a manifestly exotic state; for a recent overview see e.g. [6]. Even for the first of these unexpected states, the narrow $X(3872)$, the nature is not yet understood. A precision measurement of the line shape as it will be feasible with the PANDA experiment, however, will give a handle to sort out different models and to clarify e.g. whether it is a molecular state. Furthermore, there are important topics also in charm and open charm physics that will be adressed at PANDA, such as charmed hybrids and $D_{s}$ states.

\section{The PANDA experiment at FAIR}

One of the main pillars at FAIR that is currently under construction in Darmstadt, Germany, will be the PANDA (antiProton ANnihilation in DArmstadt) experiment dedicated to hadron physics [7]. Apart from hadron spectroscopy in the charm and light quark regions, the physics programme comprises nucleon structure, hyper nuclear and strangeness production physics, and also studies of in-medium modifications of charm in nuclear matter. The multi-purpose fixed-target PANDA 
experiment consists of a barrel detector surrounding the target region and a forward spectrometer (Fig. 1), ensuring an almost $4 \pi$ acceptance with excellent tracking, vertexing, particle identification and electromagnetic calorimetry. The PANDA experiment is directly located at the High Energy Storage Ring (HESR) that provides an antiproton beam of momenta from 1.5 to $15 \mathrm{GeV} / c$ for antiproton-proton annihilation antiproton-nucleon reactions, translating to a directly accessible invariant mass range from about 2.2 up to $5.5 \mathrm{GeV} / \mathrm{c}^{2}$.

There will be two operation modes of the HESR. The High Resolution (HR) mode optimised for beam momentum resolution will provide a beam momentum spread of $\Delta p / p=2 \times 10^{-5}$ and a luminosity of $\mathcal{L}=2 \times 10^{31} \mathrm{~cm}^{-2} \mathrm{~s}^{-1}$, whereas the High Luminosity (HL) mode will have a higher luminosity of $2 \times 10^{32} \mathrm{~cm}^{-2} \mathrm{~s}^{-1}$, while the beam momentum spread will be larger, namely $\Delta p / p=1 \times 10^{-4}$. In the initial phase, the antiproton beam is expected to be available with $\Delta p / p=5 \times 10^{-5}$ and $\mathcal{L}=1 \times 10^{31} \mathrm{~cm}^{-2} \mathrm{~s}^{-1}$. Before the accelerator will be in operation at full performance, we will have an initial phase of the experiment, PANDA Phase One (P1), for which we expect a luminosity a factor of ten lower as compared to the HL mode and a beam energy resolution about a factor of 2.5 worse as compared to HR mode, see Tab. 1.

\section{Exotic charmonium states and the role of resonance energy scans}

In $\bar{p} p$ annihilations at PANDA, hadronic states of all fermion-antifermion $J^{P C}$ quantum numbers can be produced in direct formation. This is one of the main advantages of using antiprotons as compared to $e^{+} e^{-}$annihilation, in which the hadronic system produced in direct formation is restricted to the quantum numbers of the virtual photon $\left(J^{P C}=1^{--}\right)$that acts as a spin filter.

Thanks to the cooled antiproton beam with excellent energy resolution available at PANDA, a very precise mass and width resolution can be achieved by employing the technique of an energy resonance scan (Fig. 2, b), also for resonances with $J^{P C} \neq 1^{--}$, such as the $X(3872)$ with $J^{P C}=1^{++}$. Given the small and well-controlled beam momentum spread $\Delta p / p$, the beam energy can be scanned in the centre-of-mass energy $\left(E_{\mathrm{cms}}\right)$ range around a narrow resonance, and the energy-dependent event yield can be measured. Given the beam profile is well known, it can be de-convolved, so that an energy dependent resonance cross-section measurement is obtained at high precision.

To illustrate the unique strength of PANDA in such precision line shape scans, we performed a comprehensive feasibility study [8], using the example of the famous $X(3872)$ state that is the first of the unexpected $X Y Z$ states discovered in 2003 [1], meanhwile renamed by the Particle Data Group to $\chi_{c 1}$ (3872) [9]. Theoretical interpretations come along with predictions of the decay width, however, until recently only an experimental upper limit on the absolute width of $\Gamma<1.2 \mathrm{MeV}$

Table 1: Expected beam performances for the PANDA experiment: Momentum spreads $\mathrm{d} p / p$, beam-energy resolutions $\mathrm{d} E_{\mathrm{cms}}$ and integrated luminosities $\mathcal{L}$ (at $\sqrt{s}=3.872 \mathrm{GeV}$ ) of the three different HESR operation modes, see [8] and references therein.

\begin{tabular}{c|c|c|c} 
HESR mode & $\mathrm{d} p / p$ & $\mathrm{~d} E_{\mathrm{cms}}[\mathrm{keV}]$ & $\mathcal{L}[1 /$ day $\cdot \mathrm{nb})]$ \\
\hline $\mathrm{HL}$ & $1 \cdot 10^{-4}$ & 167.8 & 13680 \\
\hline $\mathrm{HR}$ & $2 \cdot 10^{-5}$ & 33.6 & 1368 \\
\hline $\mathrm{P} 1$ & $5 \cdot 10^{-5}$ & 83.9 & 1170
\end{tabular}




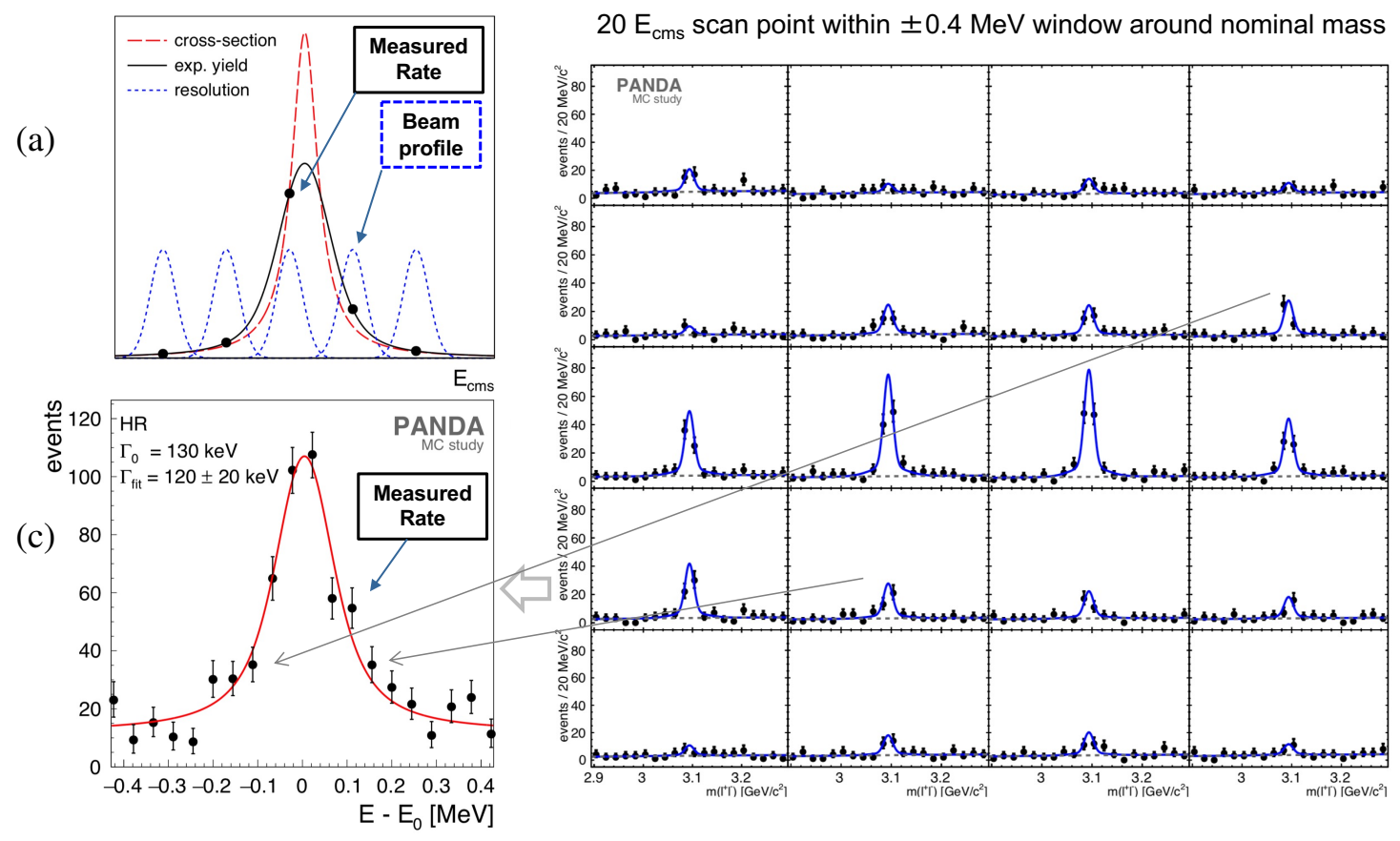

(b)

Figure 2: Illustration of a simulated MC scan experiment example $\left(\sigma_{\mathrm{s}}=100 \mathrm{nb}\right.$, Breit-Wigner width $\Gamma_{0}=130 \mathrm{keV}$, two days of data taking per $E_{\mathrm{cms}}$ position, HR mode) [8]. (a) Schematic to explain the resonance energy scan principle. (b) Simulated and reconstructed invariant dilepton spectra at each energy scan positions (going from left to right, top to bottom step-wise through the energy range $\left(E-E_{0}\right)$. (c) The resultant energy dependent event yield distribution around the nominal centre-of-mass energy $E_{0}=3.872 \mathrm{GeV}$ is fitted with a function to extract the parameter of interest, here the Breit-Wigner width $\Gamma_{0}$.
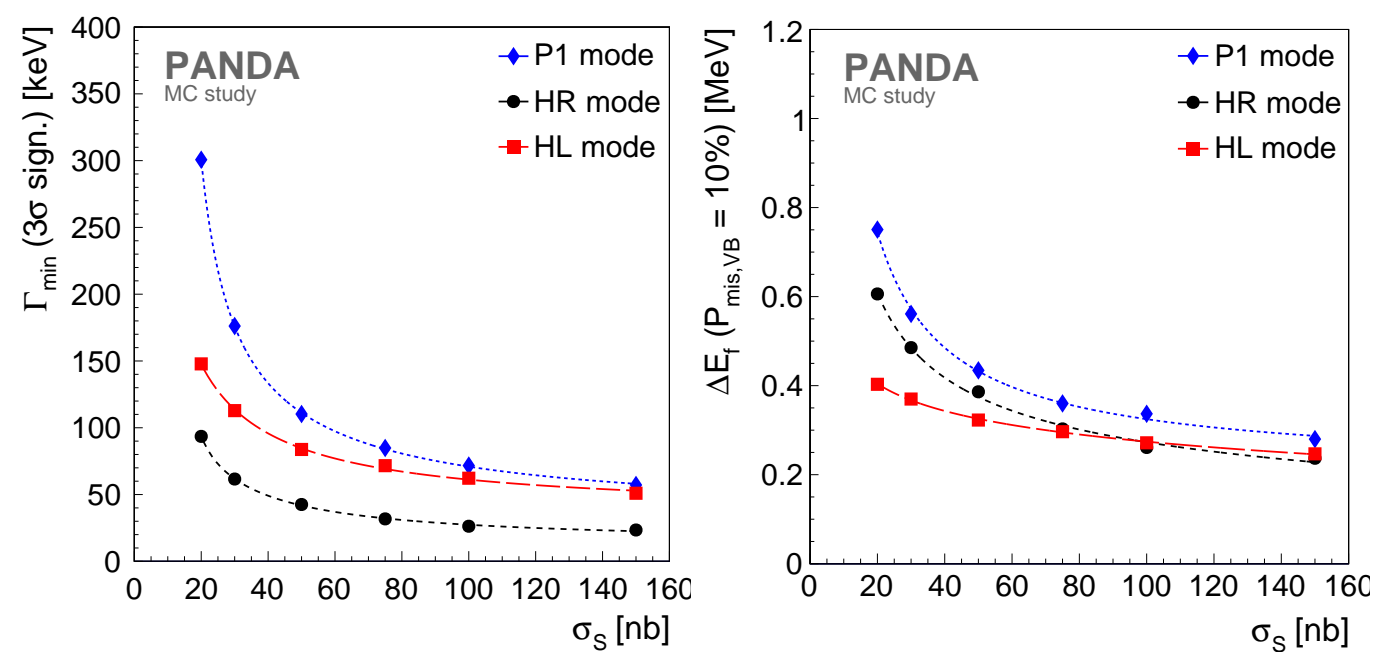

Figure 3: Summary of the sensitivity study for an absolute (Breit-Wigner) decay width measurement [8]. Left: Sensitivity in terms of the minimum decay width $\Gamma_{\min }$ that can be measured with an relative precision of $33 \%$ as a function of the assumed input $\sigma_{\mathrm{S}}$. Right: Sensitivity for line-shape measurements via the $E_{\mathrm{f}}$ parameter (Molecule case) to distinguish between a bound and a virtual state scenario in terms of the probability to mis-identify a virtual as a bound state. 

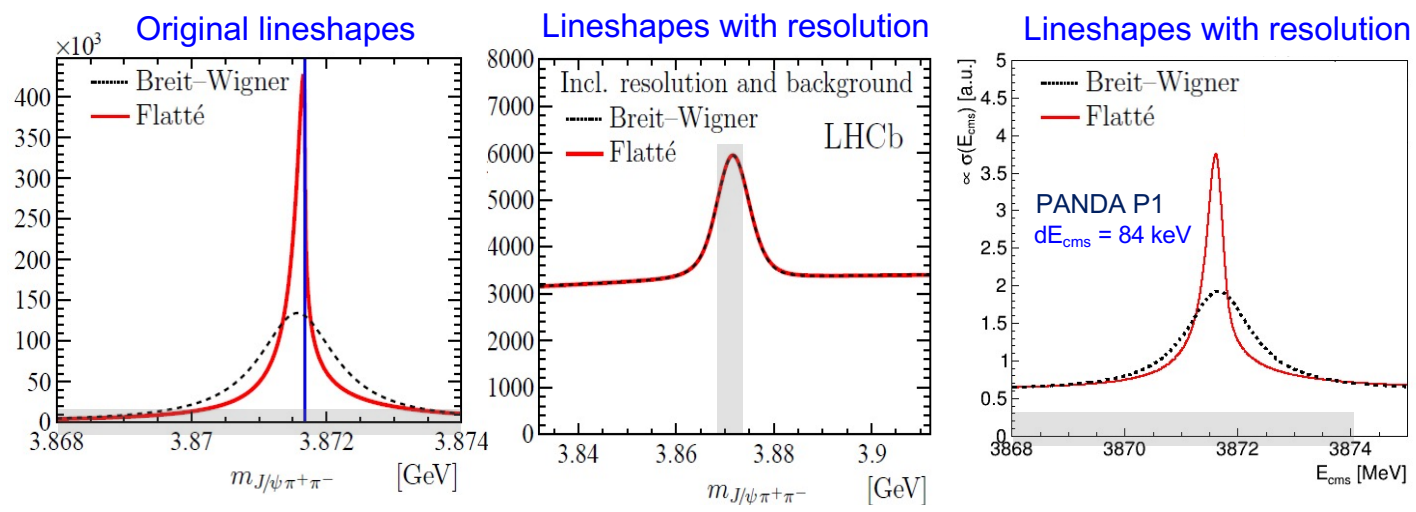

Figure 4: Comparison of the Breit-Wigner and Flatte-like line shapes without and with the LHCb and PANDA resolutions convolved. Left: The two line shapes (Breit-Wigner vs. Flatté-like) obtained from the fit to the LHCb data [13]. Centre: The same two line shapes when including backgrounds and resolution, i.e. convolved with the detector resolution. Due to the resolution, the two line shapes cannot be distinguished based on the LHCb data. Right: The same two line shapes (Breit-Wigner vs. Flatté-like) convolved with the PANDA beam energy resolution expected for the initial phase (P1) of the experiment. Thanks to the excellent beam energy resolution, they are well distinguishable with PANDA at HESR.

was provided by the Belle experiment [10]. For understanding the nature of this resonance, a precision measurement of the line shape, i.e. the energy-dependent cross section, with sub-MeV resolution has to be performed. In our MC simulation study [8], we showed the feasibilty for such a measurement with the $\bar{p} p$ annihilation experiment PANDA. We quantified the performance on the one hand for an absolute Breit-Wigner decay width measurement and on the other hand to distinguish between the bound and the virtual state scenario within a prominent molecular Flattélike line shape model via the Flatte energy paramter $E_{\mathrm{f}}$ that is directly connected with the inverse $D \bar{D}^{*}$ scattering length $[11,12]$.

The outcome of that study is summarised in Fig. 3 in terms of the minimum decay width that can be measured with a $3 \sigma$ accuracy for Breit-Wigner line shape model (Fig. 3, left) and in terms of the $10 \%$ mis-identificaton probability $P_{\text {mis }}$ to wrongly assign a virtual state as bound state (Fig. 3, left), both as a function of the assumed signal production cross section $\sigma_{\mathrm{S}}$ of the $X(3872)$. In summary, we will achieve a sub-MeV resolution for both cases, even already in the initial phase of the experiment for the accelerator operation mode P1 within 80 days of data taking.

Since recently, a first absolute decay width measurement of $\Gamma_{\mathrm{BW}}=1.39 \mathrm{MeV}$ has been published by the LHCb Collaboration based on their data. The observed signal in the LHCb data is equally well described by both models, and thus they can not distinguish between a Flatté-like and a Breit-Wigner line shape [13]. The line shapes as obtained by fits to the LHCb data are shown in Fig. 4 (left). When convolved with the resolution, the two line shapes are indistinguishable at $\mathrm{LHCb}$ (Fig. 4 centre). It is due to the detector resolution that the data is equally well described when using the two corresponding line shape models. The same two line shapes convolved with the PANDA beam energy resolution as expected for the initial phase of the experiment, namely for the accelerator operation mode P1, are displayed in Fig. 4 (right). Thanks to the excellent beam energy resolution, they will be well distinguishable with PANDA at HESR. 

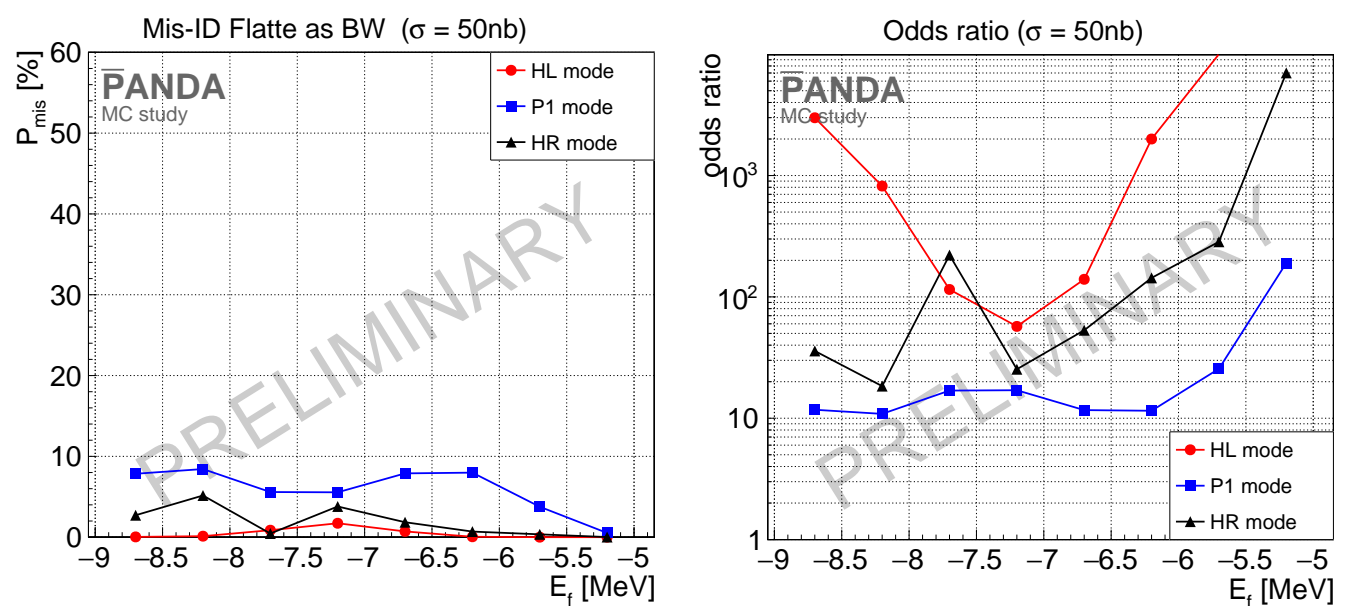

Figure 5: Left: Sensitivity in terms of the mis-identification probability $P_{\text {mis }}$ to wrongly assign the BreitWigner line shape instead of the correct Flatté-like line shape as a function of the Flatté energy parameter $E_{\mathrm{f}}$, whereas $P_{\text {mis }}=50 \%$ corresponds to "indistinguishable". Right: The correspondingly computed so-called "odds", i.e. the number of correct assignments per wrong one, defined as $o d d s:=\left(1-P_{\text {mis }}\right) / P_{\text {mis }}$. Using this measure, the expected performnace is at least ten times better than "indistinguishable"

As an addendum to our published sensitivity study [8], we investigated and quantified meanwhile the expected PANDA performance in distinguishing these two different line shape models in addition. The assumptions and ingredients we used in the corresponding simulations are the same as we employed in [8], whereas this study is now restricted to an assumed signal production cross section of $\sigma_{S}=50 \mathrm{nb}$. We investigated a larger range of line shape parameters for the two different models, namely 10 different Breit Wigner decay widths $\Gamma$ and 8 different Flatté energies $E_{\mathrm{f}}$. For each setting of input parameters, $10 \mathrm{k}$ data spectra have been generated, to each of which we fitted both models with the parameters left free floating in the fit. And for each input parameter setting, we compared the fit qualities between the best fits of either model to a given spectrum. Whenever the model used for generation of the spectrum has led to a worse fit probability than the competing one, the case has been counted as a mis-identification.

The achievable performance has been evaluated in terms of the mis-identification probability $P_{\text {mis }}$ to assign the wrong line shape model, namely the Breit-Wigner line shape for the simulated MC data generated using the Flatté line shape, and vice versa. More details have recently been discussed at QWG2021 and can be found in the presented slides [14].

The outcome is summarised in Fig. 5. The resultant sensitivities in assigning the correct line shape (shown here for the Flatté-like line shape) are better than $90 \%$ to $98 \%$ (and the results are practically similar for the Breit-Wigner line shape wrongly assigned to be the Flatté-like), depending on the given accelerator operation mode (Fig. 5, left). It should be noted that for this figure of merit, a mis-identification probability of $P_{\text {mis }}=50 \%$ corresponds to "indistinguishable". In order to answer the question, how much better our expected performance is as compared to "indistingusihable", one may consider the so-called "odds" defined as the number of correct assignments per wrong one: odds $:=\left(1-P_{\text {mis }}\right) / P_{\text {mis }}$. The corresponding odds computed for the results of our study are shown in Fig. 5, (left). Using this measure, with PANDA, we expect to be at least a factor of 10 better than "indistinguishable". 

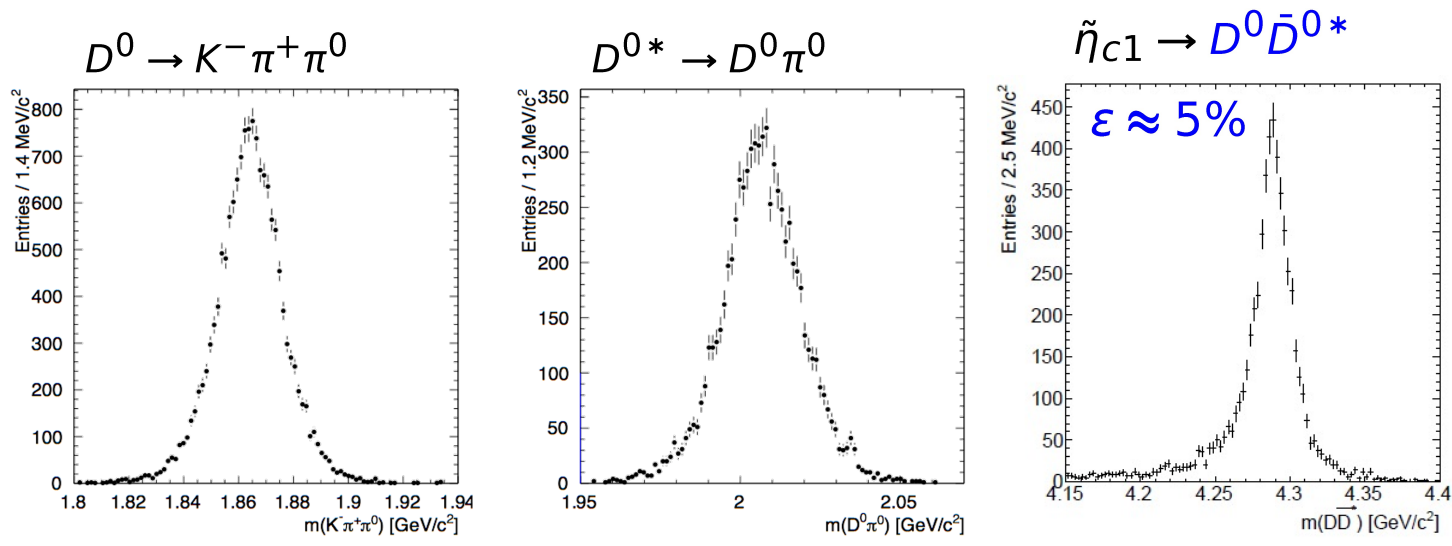

Figure 6: Feasibility study for the open charm decay of the lowest lying spin-exotic charmed hybrid candidate $\tilde{\eta}_{\mathrm{c} 1}$ at PANDA in the reaction $p \bar{p} \rightarrow \tilde{\eta}_{\mathrm{c} 1} \eta$, with $\tilde{\eta}_{\mathrm{c} 1} \rightarrow D^{0} \bar{D}^{0^{*}} \pi^{0}$. Shown are the different stages of reconstructed invariant masses [7]. Left: Reconstructed invariant mass of $D^{0} \rightarrow K^{-} \pi^{+} \pi^{0}$. Centre: Reconstructed invariant mass of $D^{0 *} \rightarrow D^{0, *} \pi^{0}$. Right: Reconstrcuted invariant mass of $\tilde{\eta}_{\mathrm{c} 1} \rightarrow D^{0} \bar{D}^{0^{*}} \pi^{0}$.

\section{Charmed hybrids}

The charmonium region is also well suited to search for charmed hybrid states. Thanks to the lower level density as compared to the light meson sector and the charmonium states being more narrow, resulting in less overlaps, interferences are less severe, and thus the identification of exotic hybrid states is easier. Especially the search for spin-exotic hybrid states with non- $q \bar{q}$ quantum numbers is promising as they do not mix with ordinary mesons. The lowest lying hybrid state in the charmonium region with exotic quantum numbers $J^{P C}=1^{-+}$is predicted e.g. by Lattice QCD [15] and also by newer calculations [16] at a mass of about $4.3 \mathrm{GeV} / c^{2}$, the so-called $\tilde{\eta}_{\mathrm{c} 1}$.

Based on Lattice QCD, one preferred decay channel is to $c \bar{c}$ [P-wave] plus $\pi \pi$ [S-wave], means for example $\tilde{\eta}_{\mathrm{c} 1} \rightarrow \chi_{\mathrm{c} 1} \pi^{0} \pi^{0}$ or $\tilde{\eta}_{\mathrm{c} 1} \rightarrow D^{0}{\overline{D^{0}}}^{0^{*}} \pi^{0}$. Both final states need good electromagnetic calorimetry and the open charm deacy in addition good particle identification, i.e. pion-to-kaon separation. Assuming a signal production cross section for $p \bar{p} \rightarrow \tilde{\eta}_{\mathrm{c} 1} \eta$ similar to $p \bar{p} \rightarrow \psi(2 S) \eta$ of $33 \mathrm{pb}$, a feasibility study was performed for these two favoured decay channels with the PANDA experiment [7]. For the hidden charm decay, a reconstruction efficiency of about $7 \%$ was estimated. Since for the signal over background ratio $S / B>250 \cdot \sigma_{\mathrm{S}} / \sigma_{\mathrm{B}}$ was obtained, this channel is expected to be well feasible for signal and background production cross sections fulfilling $\sigma_{\mathrm{B}}<10 \cdot \sigma_{\mathrm{S}}$. Similarly for the open charm decay, for which a detection efficiency of about $5 \%$ was estimated (Fig. 6), this channel will be feasible at PANDA for any non-vanishing branching ratio [7]. 


\section{Open charm topics}

There are many interesting open charm topics to be adressed by the PANDA experiment, particularly on $D_{s}$ states. In general there is good qualitative agreement for the $D_{s}$ spectrum between theory and experiment. Apart from some new $D_{\mathrm{J}}$ states reported by $\mathrm{LHCb}$, there are the two narrow states $D_{s 0}^{*}(2317)$ and $D_{s 1}^{*}(2460)$ still under discussion, since they have masses lower than expected, just beneath the $D K$ and $D K^{*}$ mass thresholds. Since the decay widths have not yet been measured, the nature of these states is unclear.

Even though it is not possible to produce the $D_{s 0}^{*}(2317)$ in direct formation at PANDA, simply for the reason that it is charged, one may consider an energy scan with recoil particle at threshold. One important condition for this purpose is that the used recoil has to be rather narrow (or to be of a very precisely known width), which is fulfilled for the $D_{s}^{+}$. The cross section for the reaction $p \bar{p} \rightarrow D_{s}^{+} D_{s 0}^{*}(2317)$ and the line shape at threshold depend on the decay width $\Gamma$ of the $D_{s 0}^{*}(2317)$. The width can be accessed via the so-called excitation function, which connects the cross section and the width. A feasibility study for such an energy scan at threshold has been carried out for the PANDA experiment [7]. The MC simulation study was based on $40 \mathrm{k}$ signal and $10 \mathrm{M}$ generic background events simulated with an antiproton beam momentum of $p_{\text {beam }}=8.8 \mathrm{GeV} / c$. The $D_{s}^{+}$is inclusively reconstructed, employing the missing mass technique, and results in a detection efficiency of about $25 \%$. The simulated energy scan at threshold is illustrated in Fig. 7, showing 15

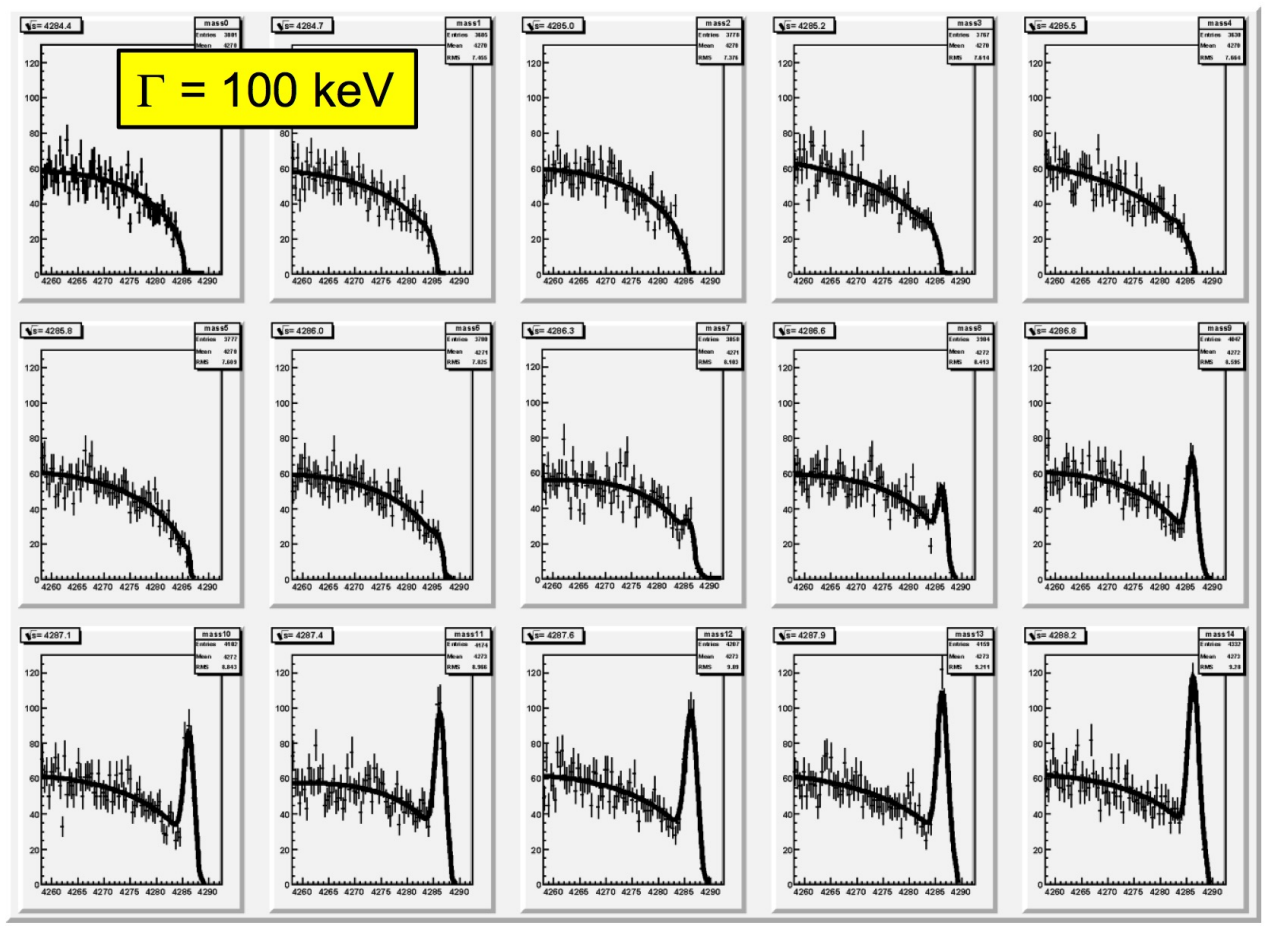

Figure 7: Illustration of the simulated energy scan at threshold of the reaction $p \bar{p} \rightarrow D_{s}^{+} D_{s 0}^{*}$ (2317) to measure the excitation function in order to determine the decay width of the $D_{s 0}^{*}(2317)$. Shown are the measured and reconstructed invariant mass spectra at 15 energy scan points. The assumed input decay width is $100 \mathrm{keV}$ [17]. 

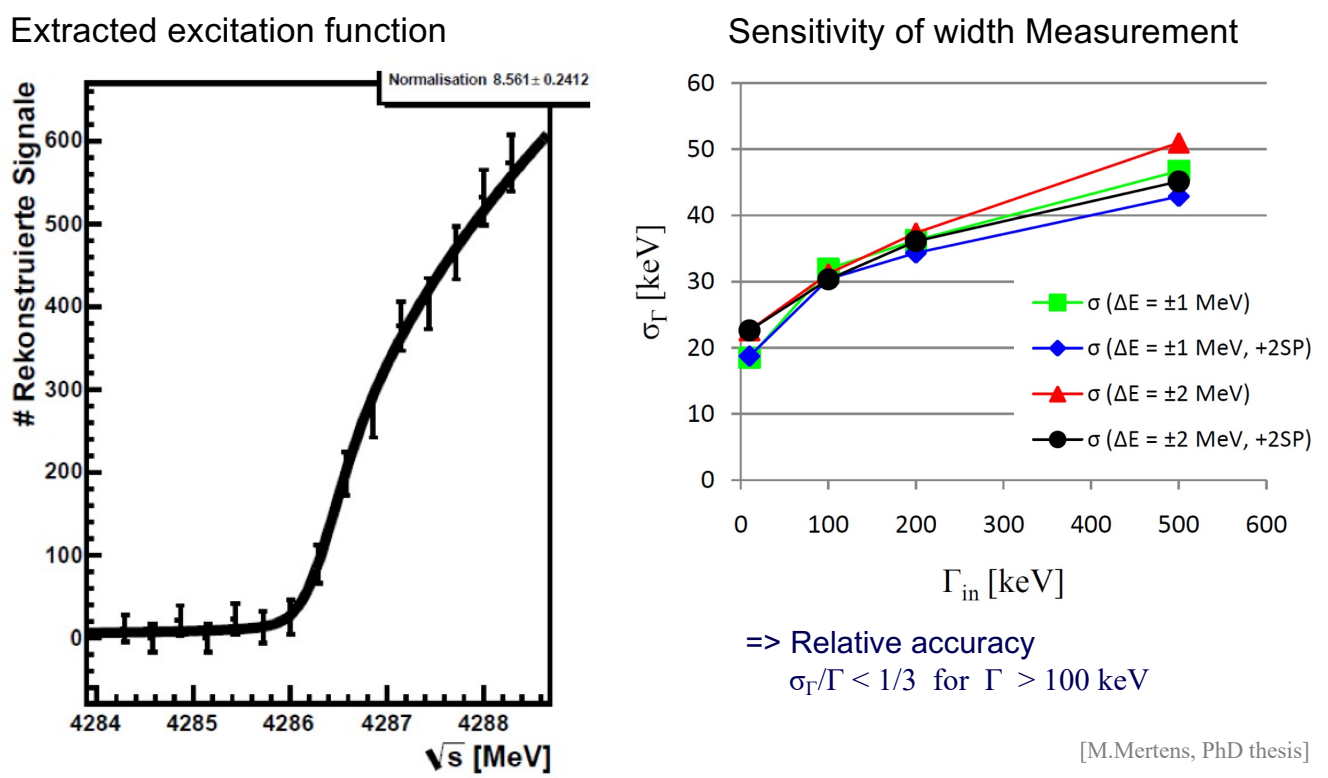

[M.Mertens, $\mathrm{PhD}$ thesis]

Figure 8: Results of a simulated threshold scan with recoil. Left: Plotted is the number of extracted signal events as a function of centre-of-mass energy. Right: Sensitivity of the width measurement in terms of the error $\sigma_{\Gamma}$ of the measured width as a function of the assumed input decay width $\Gamma_{\text {in }}$ of the $D_{s 0}^{*}(2317)$ [17].

energy scan points within a $4 \mathrm{MeV}$ window around the mass of the $D_{s 0}^{*}(2317)$. An decay width of $\Gamma\left(D_{s 0}^{*}(2317)\right)=100 \mathrm{keV}$ was assumed.

The number of signal events as extracted by the fits to the 15 reconstructed mass spectra (Fig. 7) are shown as a function of the centre-of-mass energy together with the fitted excitation function in Fig. 8 (left). The outcome of that study in terms of the sensitivity for such a width measurement is summarised in Fig. 8 (right). In summary, we expect a relative accuracy of $\sigma_{\Gamma} / \Gamma_{\text {in }}<1 / 3$ for input widths larger than $100 \mathrm{keV}$.

\section{Summary and conclusions}

The PANDA experiment under construction at FAIR in Darmstadt, Germany, will be the facility to study QCD in Europe. The broad physics programme comprises among others hot topics in hadron spectroscopy, especially in the charmonium region. The possibility of resonance energy scans with the antiproton beam of excellent energy resolution offers unique opportunities for both, topics in charmonium as well as open charm physics.

By a comprehensive MC sensitivity study for such energy scans of narrow states, the achievable performances for precision measurements of absolute decay widths and line shapes have been quantified for the example of the famous charmonium-like exotic $X$ (3872) state. The sensitivities have been studied for three anticipated HESR accelerator operation modes (P1, HL, HR). Already in the initial phase P1 of the experiment, PANDA will reach sub-MeV resolutions. Moreover, a proof principle for an experimental distinction between different line shapes for the $X(3872)$ has been provided. The sensitivity to distinguish between a Flatté-like and a Breit-Wigner line shape, 
which appear indistinguishable based on the LHCb data, has been quantified to be at least ten times better then indistinguishable, again already for the initial phase P1, $c f$. also [18].

Employing an energy scan at threshold with recoil particle, also the sensitivity for a measurement of the decay widths of the $D_{s 0}^{*}(2317)$ has already been quantified in a first MC simulation study. Also here, we expect to achieve sub-MeV resolution for the absolute decay width measurement, as needed to better understand the nature of the narrow $D_{s 0}^{*}(2317)$ state. Furthermore, PANDA will be well suited to search for and study charmed hybrid states in the charmonium region, such as the lowest lying spin-exotic hybrid candidate $\tilde{\eta}_{\mathrm{c} 1}$ with $J^{P C}=1^{-+}$predicted at a mass of about $4.3 \mathrm{GeV} / c^{2}$.

\section{References}

[1] S.K. Choi et al. (Belle Collaboration), Phys. Rev. Lett. 91, 262001 (2003).

[2] B. Aubert et al. (BaBar Collaboration), Phys. Rev. Lett. 95, 142001 (2005).

[3] B. Aubert et al. (BaBar Collaboration), Phys. Rev. Lett. 98, 212001 (2007).

[4] M. Ablikin et al. (BESIII Collaboration), Phys. Rev. Lett. 110, 252001 (2013).

[5] Z.Q. Liu et al. (Belle Collaboration), Phys. Rev. Lett. 110, 252002 (2013).

[6] R.F. Lebedev, R.E. Mitchell and E.S. Swanson, arXiv:1610.04528v2 [hep-ex].

[7] The PANDA Collaboration, arXiv:0903.3905 [hep-ex].

[8] G. Barucca et al. (PANDA Collaboration), Eur. Phys. J. A 55, 42 (2019).

[9] https://pdglive.lbl.gov

[10] S.-K. Choi et al. (Belle Collaboration), Phys. Rev. D 84, 052004 (2011).

[11] C. Hanhart et al., Phys. Rev. D 76, 034007 (2007).

[12] Yu. S. Kalashnikova et al., Phys. Atom. Nucl. 73, 1592-1611 (2010).

[13] R. Aaij et al. (LHCb Collaboration), Phys. Rev. D 102, 092005 (2020).

[14] K. Götzen and F. Nerling, for the PANDA Collaboration, "Can we resolve the nature of the $\chi_{\mathrm{c} 1}(3872)$ ?”, 14th Intern. Workshop on Heavy Quarkonium, 15-19th March 2021 (2021).

[15] G.S.-Bali, Int. J. Mod. Phys. A21, 5610 (2006).

[16] N.-Brambilla et al., Phys. Re. D 99, 014017 (2018).

[17] M. Mertens, PANDA PhD thesis (2015).

[18] G. Barucca et al. (PANDA Collaboration), Eur. Phys. J. A 57, 184 (2021). 